\title{
GAMIFICAÇÃO: SUA APLICAÇÃO NA EDUCAÇÃO E AS IMPLICAÇÕES PARA O CONTEXTO DO ENSINO DE ENGENHARIA
}

\author{
GAMIFICATION: ITS APPLICATION IN EDUCATION AND THE IMPLICATIONS FOR THE \\ CONTEXT OF TEACHING OF ENGINEERING \\ Lucas Bergman Aleixo Fósculo de Moura ${ }^{1}$, Leonardo Assírio \\ de Oliveira Bossi², Paula Karina Salume ${ }^{3}$ \\ DOI: 10.37702/REE2236-0158.v40p128-136.2021
}

\begin{abstract}
RESUMO
Novas tendências e desafios para a educação têm sido discutidos a partir do maior entendimento do processo de aprendizado como resultado de uma construção social e da popularização de tecnologias, capazes de proporcionar maior interação e colaboração entre pessoas. Nesse contexto, o uso da gamificação, como alternativa que permite aperfeiçoar aspectos como motivação e engajamento, também tem sido apontado como uma das principais tendências para a educação para os próximos anos. Assim sendo, o objetivo geral deste artigo é discutir a aplicação de modelos combinados e da gamificação como forma de potencializar o engajamento e a motivação de estudantes, além de auxiliar a metodologia tradicional de ensino a superar os desafios do ensino em Engenharia de Produção. Para atingir o objetivo proposto, o presente trabalho explora três pontos principais: o caso da escola americana Quest to Learn, a primeira no mundo com um currículo inteiramente baseado em princípios da gamificação; a aplicação de jogos no Ensino de Engenharia para estudantes do ensino superior; e o framework genérico de gamificação em contextos educacionais proposto por Silva e Bax (2017), avaliando seu potencial de utilização no Ensino em Engenharia.
\end{abstract}

Palavras-chave: tendências na educação; Ensino em Engenharia; gamificação.

\begin{abstract}
New trends and challenges in higher education have been discussed for a better understanding of learning as a result of social construction and the popularization of technologies, capable of providing greater interaction and collaboration among people. In this context, the use of gamification as an alternative to improve some aspects such as motivation and engagement has also been pointed out as one of the main trends in higher education for the next years. Therefore, the main goal of this study is to discuss the application of blended models of learning and gamification as an alternative to improve the engagement and motivation of students, as well as assist the conventional teaching proposal to overcome the new challenges that the teaching of Industrial Engineering has been facing. To fulfill this aim, this article focus on three main points: the case of an American school named Quest to Learn, the first one with a curriculum entirely based on gamified principles; the application of games in teaching of engineering for higher degree students; and the generic framework of gamification in educational contexts proposed by Silva and Bax (2017), as well as evaluating its potential in teaching of engineering.
\end{abstract}

Keywords: trends in education; teaching of engineering; gamification.

\footnotetext{
${ }^{1}$ Engenheiro de Produção formado na PUC-MG; lucasbergmanmoura@gmail.com

${ }^{2}$ Engenheiro de Produção formado na PUC-MG; leoassirio@gmail.com

${ }^{3}$ Professora do Departamento de Ciências Administrativas e Contábeis da Universidade Federal de São João Del-Rei. paulasalume@hotmail.com
} 


\section{INTRODUÇÃO}

Azevedo e Gontijo (2017) apontam o desenvolvimento de competências e o fomento do desenvolvimento contínuo de ações por parte dos estudantes como os principais desafios do curso de Engenharia de Produção no Brasil. Segundo os autores, a formação deste profissional enfrenta contínuas transformações devido ao avanço e emprego de novas tecnologias. Adicionalmente, por se tratar de um curso relativamente novo, inserido no mercado brasileiro há poucas décadas, torna-se bastante significativo o estudo de metodologias e abordagens que auxiliem o desenvolvimento de competências do engenheiro de produção e que, paralelamente, contribuam para a adaptação da formação profissional deste frente ao cenário de constante adoção de novas tecnologias.

Nesse contexto, Becker et al. (2017) também apresentam em Horizon Report: 2017 Higher Education Edition os principais pontos relacionados às tendências e tecnologias para a educação superior nos próximos anos. No relatório consta que o aprendizado colaborativo e o uso de modelos combinados de aprendizagem são os principais impulsionadores do uso da tecnologia na educação para os próximos anos. Segundo os autores, o aprendizado colaborativo pode ser entendido como o trabalho realizado em conjunto entre estudantes e educadores, seja em atividades em grupo ou em pares. Além de promover engajamento e o sentimento de consecução entre os estudantes, esse tipo de aprendizado fomenta o compartilhamento de informações e experiências, capazes de influenciar diretamente no desempenho dos envolvidos. Outras tendências, exploradas por Becker et al. (2016), estão relacionadas com o foco crescente nas medições do aprendizado e ao uso de gamificação como alternativa que permite coletar dados sobre o desempenho e engajamento dos alunos.

Kapp (2012) define gamificação como "o uso de mecânicas, estéticas e pensamentos dos games para engajar pessoas, motivar a ação, promover a aprendizagem e resolver problemas" (KAPP, 2012, p. 10) e aponta a educação como uma das áreas com maior potencial de aplicação. Hamari, Koivisto e Sarsa (2014) defendem a gamificação como um tema relativamente novo no âmbito acadêmico e com poucas estruturas teóricas bem estabelecidas. Sailer et al. (2017) associam a gamificação à inovação e evidenciam também como o universo acadêmico enxerga o tema: um processo promissor e aplicável a uma grande variedade de contextos. Fortunato e Teichner (2015) expandem a possibilidade da gamificação na educação, relacionando-a diretamente com a afinidade dos jovens do século XXI com os videogames. Segundo os autores, ignorar a capacidade dos jogos de cativar os jovens e não os aplicar no processo de ensino-aprendizagem seria um desperdício. Nesse contexto, Lemes e Sanches (2016) introduzem a Quest to Learn, uma escola pública norte-americana pioneira na utilização da gamificação. Por meio de um ensino inteiramente baseado em princípios de jogos, a escola modificou a experiência dos estudantes e atingiu resultados expressivos em relação a engajamento e desempenho.

Ao compreender a gamificação como um processo que busca tornar atividades de contextos non-game adaptadas a games, utilizando elementos de design de jogos, Sailer et al. (2017) retratam que o processo pode tomar diferentes formas e combinar diferentes elementos, de diversas maneiras, sendo inapropriado estudar os efeitos motivacionais da gamificação como algo genérico. No cenário educacional, é de fundamental importância a identificação de um modelo ótimo para esse processo, que motive e engaje o aluno no aprendizado. Dessa forma, Silva e Bax (2017) citam uma lacuna relevante de pesquisa nos modelos de gamificação propostos para fomentar a aprendizagem colaborativa. Segundo os autores, aplicar mecânicas de jogos sem considerar os diferentes perfis de usuários pode resultar em modelos de sistemas gamificados incompletos, que não atingirão de forma mais ampla os objetivos de aprendizagem.

Tendo isso exposto, este artigo pretende investigar se a aplicação de modelos combinados e a utilização da gamificação podem potencializar o engajamento e a 
motivação de estudantes, incluindo os estudantes de Engenharia de Produção no Brasil, fomentando o desenvolvimento contínuo de suas ações, assim como se permitem gerar maior capacidade de adaptação ao ensino para as instituições que utilizam dessas ferramentas. Mais especificamente, buscou-se explorar os princípios e resultados de uma escola norte-americana que aplicou a gamificação na estruturação do seu currículo e metodologia de ensino, assim como nortear e elucidar a aplicação de jogos no ensino superior e os principais resultados dessa aplicação.

\section{DESENVOLVIMENTO}

Nesta seção é apresentada uma breve revisão sobre o contexto atual da Engenharia de Produção no Brasil, os principais conceitos relacionados à gamificação e sua aplicação, com exemplos no contexto educacional. A primeira seção retrata o atual cenário brasileiro e os desafios da "Educação em Engenharia de Produção". A segunda seção analisa brevemente alguns exemplos de jogos aplicados no Ensino de Engenharia, como forma de ampliar o estudo de gamificação no contexto educacional do ensino superior. A terceira seção, por sua vez, apresenta o conceito de "gamificação" e discute o uso da gamificação na educação, utilizando-se como referência a escola americana Quest to Learn. Por fim, na quarta seção, buscou-se identificar na literatura algum modelo que auxilie no entendimento dos aspectos e aplicação de práticas gamificadas no contexto educacional, sendo escolhido o trabalho realizado por Silva e Bax (2017).

\section{O Ensino em Engenharia de Produção}

Azevedo e Gontijo (2017) refletem sobre o atual cenário do tema "Educação em Engenharia de Produção". Conforme constatado pelos autores, o Brasil ocupou em 2017 a $19^{a}$ posição no ranking mundial de publicações sobre o tema. Com apenas $1 \%$ do total de publicações globais, é evidente a necessidade de uma maior exploração desse tema para que o perfil do profissional da Engenharia de Produção seja mais bem compreendido. Adicionalmente, a carência de estudos dificulta a adaptação da formação profissional do Engenheiro de Produção, uma vez que o ensino de Engenharia de Produção enfrenta o contínuo desenvolvimento e inserção de novas tecnologias. Frente a esse cenário, as instituições são desafiadas a aplicar metodologias que fomentem o desenvolvimento contínuo de ações por parte dos estudantes (AZEVEDO; GONTIJO, 2017).

De forma complementar, Silva et al. (2016) destacam como o Ensino de Engenharia de Produção requer uma forte aproximação com o mundo real. Para que essa aproximação ocorra na prática, metodologias de aprendizagem vivencial aparecem como tendências que complementam o aprendizado tradicional e fomentam o desenvolvimento de competências por parte dos estudantes. De acordo com os autores, na aprendizagem vivencial o discente passar a ser o centro do processo, facilitando um envolvimento maior por parte do estudante. Como apresentado no artigo, no caso específico do Ensino em Engenharia de Produção, jogos, simulações e dinâmicas vêm constantemente sendo utilizados como metodologias de aprendizagem vivencial que permitem uma aplicação mais concreta de conceitos abstratos ligados aos campos de planejamento e controle da produção, assim como para o desenvolvimento de competências relacionadas às respectivas áreas, tais como trabalho em equipe, resolução de conflitos e capacidade de vivenciar ambientes que apresentam algum grau de incerteza.

\section{Jogos no Ensino de Engenharia}

Jogos com metodologias que simulam situações reais e demandam de seus jogadores um pensamento lógico e ações contínuas para solucionar os problemas propostos acabam desenvolvendo em seus participantes conhecimento e prática os quais o ensino tradicional pode não alcançar com a mesma eficiência em um mesmo intervalo de tempo. Nesse sentido, Kafai (2001) cita o histórico da utilização dos mundos virtuais como forma de propiciar jogos e simulações para facilitar o processo de aprendizagem. 
Identificou-se três jogos com aplicação da gamificação no Ensino da Engenharia. São eles: Scratch $₫$, Production Line $®$ e Kanban S.A.

O Scratch ${ }^{\circledR}$ foi criado pelo Lifelong Kindergarten Group, do Massachusetts Institute of Technology (MIT), que, em parceria com o grupo KIDS da Universidade da Califórnia, buscava uma alternativa para oferecer um software que ensinasse, a partir da brincadeira, linguagem de programação. Inicialmente concebido para alunos do Ensino Fundamental, a estratégia lúdica e visual da ferramenta permitiu sua adoção no ensino superior de diversos países do mundo, assim como por pesquisadores nas áreas de tecnologia e programação. O resultado foi tão positivo que, atualmente, tenta-se associar também projetos de inteligência artificial, redes neurais, deep learning, entendimento e tradução de textos, jogos de nicho entre outros (MALAN; LEITNER, 2007).

Por sua vez, o Production Line®, lançado pela Positech Games em 2017, coloca o jogador na posição de um presidente de uma companhia automotiva, sendo este o responsável por projetar a sua fábrica e lucrar com a venda de seus carros produzidos. Existem mais de 42 tipos de máquinas e mais de 150 tipos de matéria-prima, sob os quais o jogador deve tomar decisões e analisar os resultados a cada rodada. Além de produzir carros, os participantes também devem tomar decisões relacionadas à matéria-prima, por exemplo, optando por produzi-la localmente ou importála. Dessa forma, há um intenso aprendizado sobre o balanceamento de recursos, assim como sobre o processo de tomada de decisão, sobre a avaliação das consequências positivas e negativas, sobre gestão financeira e de campanhas de marketing, com forte relação com a prática no mundo real. Apesar de não terem sido encontrados estudos sobre a utilização desse jogo no ensino superior, fica evidente seu potencial de aplicação, principalmente para estudantes de Engenharia de Produção. Há, basicamente, a abordagem de diversos conceitos relevantes para a formação de um engenheiro: produtividade, lead time, tempo de importação, porcentagem de recurso ocioso, layout de fábrica, etc.
Por fim, o Kanban S.A., desenvolvido pelo engenheiro de produção Filipe Carvalho, tem como principal abordagem induzir o pensamento crítico sobre a gestão da produção, colocando o jogador dentro de um cenário industrial e de empreendedorismo, em que ele se torna responsável por gerir seus recursos e ampliar seu lucro. A cada rodada, os jogadores utilizam seus colaboradores para transformar sua matéria-prima disponível em produto final, tendo que trabalhar aspectos como gestão de estoque, gestão de riscos e gestão financeira. Apesar de tanto a aquisição da matéria-prima quanto a venda do produto final estarem ligadas à sorte do jogador com os dados, o tabuleiro é organizado de forma que se simula um ambiente incerto, representando as incertezas e desafios presentes no mercado.

Com a aplicação o jogo em várias turmas do curso de Engenharia de Produção, pôde-se observar que o game contribuiu tanto para a apresentação de novos conteúdos quanto como para a fixação destes. Adicionalmente, verificou-se uma forte relação entre diversão e motivação para o aprendizado, um dos principais aspectos abordados pela gamificação (CARVALHO; CUNHA JÚNIOR; OLIVEIRA, 2019).

\section{Gamificação e sua Aplicação no Contexto Educacional}

Kapp (2012) define gamificação como o uso de mecânicas, estéticas e pensamentos dos games com o propósito de engajar pessoas, motivar a ação, promover a aprendizagem e resolver problemas. É a junção de todos esses elementos - mecânica, estética, pensamento, engajamento, aprendizagem e resolução de problemas - que cria uma experiência capaz de envolver os participantes.

Em seu trabalho, Kapp (2012) também explora cada um dos aspectos integrantes de sua definição de gamificação. Segundo ele, a gamificação se relaciona com os games à medida que cria um sistema colaborativo, em que os gamers, aprendizes, consumidores e proponentes de um determinado desafio interagem e se relacionam entre si na busca por uma solução mais adequada. Esse aspecto de 
jogo faz com que os envolvidos queiram investir conhecimento, tempo e energia, promovendo um ambiente de interação, desafio e trocas de experiências positivas para todos. Outros aspectos que ganham destaque na visão de Kapp (2012) são a mecânica e o pensamento de jogos. A mecânica caracteriza o aspecto de jogo da experiência, atribuindo níveis, sistemas de pontuação e bonificação e restrições. Já o pensamento de jogos (Game Thinking), tido como o principal elemento da gamificação, está relacionado com a ideia de pensar um evento cotidiano, como caminhar ou correr, em uma atividade narrativa com elementos de competição, cooperação e que pode ser explorada de alguma forma. Como exemplo, o autor cita a corrida como um processo social, em que amigos competiriam uns contra os outros, encorajando-se em um ambiente colaborativo, enquanto um corredor conta a história de correr algumas centenas de milhas ou de escapar de zumbis.

$\mathrm{O}$ autor também explica o motivo de a gamificação fomentar o aprendizado: muitos elementos da gamificação são baseados na psicologia da educação, com técnicas usadas há anos por professores e instrutores. Por fim, outro aspecto que merece destaque é a reflexão proposta pelo autor sobre o que não é gamificação. Segundo ele, a gamificação não deve ser resumida a atribuição de pontos, recompensas ou níveis. Os aspectos dos games que realmente caracterizam a experiência gamificada são a narrativa, engajamento, visualização e entendimento dos papéis e a busca pela resolução de problemas.

Kaufmann (2018) destaca que o principal objetivo da gamificação é fomentar o aprendizado e que, para o processo funcionar, o aluno deve estar motivado e engajado no aprendizado. Para o autor, o objetivo da gamificação é atingido apenas dessa forma. Dicheva et al. (2015) consideram que a aplicação de uma certa combinação de princípios e de elementos - por exemplo pontos, tabelas de líderes, barras de progresso e desafios - levará a resultados motivacionais específicos. McGonigal (2011) avalia a motivação do usuário e sua escolha pelo game ao invés de realizar outra atividade ou, até mesmo, uma responsabilidade: segundo a autora, essa pessoa provavelmente deseja escapar da pressão ou monotonia relacionada à atividade. McGonigal também constata a tendência desse trade-off entre game $\mathrm{e}$ responsabilidades e explica que essa tendência se deve a razões específicas, sendo, em muitos casos, a diversão a principal delas.

No contexto do ensino fundamental e do ensino médio, de acordo com McGonigal (2011), os games estão sendo utilizados para reinventar a educação pública usualmente conhecida. A autora dá grande destaque à escola pública Quest to Learn, localizada em Nova Iorque, nos Estados Unidos. Fundada em 2009, é a primeira escola do mundo com um currículo inteiramente baseado em jogos e em princípios da gamificação, tendo como objetivo principal oferecer uma realidade alternativa para os alunos durante o aprendizado. McGonigal (2011) defende o modelo de aprendizado leveling up proposto pela escola e alega que o sistema é um modelo de sucesso mais igualitário que o sistema tradicional de avaliação. Dessa forma, todos os alunos podem sempre buscar níveis mais altos desde que continuem trabalhando forte em busca do principal objetivo da educação: o aprendizado. O modelo leveling up, também conhecido como modelo de nivelamento, pode substituir, ou até mesmo complementar, o modelo tradicional de avaliação e aprendizado baseado em notas de avaliações e apenas uma chance de ganhar. Lemes e Sanches (2016) evidenciam os fatores que motivaram a Quest to Learn a buscar uma nova proposta de aprendizado. Como constatado pelos autores, o modelo tradicional de ensino não mostrava uma solução clara que combatesse o cenário de alta taxa de desistência no ensino médio nos Estados Unidos. A falta de engajamento e motivação que os alunos têm em relação à escola eram as principais razões de tal cenário. Por meio de dados obtidos pelos autores junto à instituição Quest to Learn, evidenciou-se a dimensão do problema. O engajamento que começa alto no ensino infantil, chegando a $80 \%$, cai para $60 \%$ no ensino fundamental, $40 \%$ no ensino médio e para $30 \%$ no ensino superior. Sendo o engajamento e a motivação dois grandes resultados gerados pela gamificação, constatouse uma grande oportunidade para resolver as 
dores dos alunos no processo de ensino por meio da gamificação.

Por meio da definição de princípios a serem seguidos pela instituição, apresentados no Quadro 1, e de posterior aplicação de um currículo diferenciado que alinhe objetivos e metodologia de ensino, a escola pública novaiorquina atingiu conquistas relevantes: aumento da frequência escolar; fixação do corpo de professores, uma vez que os mesmos queriam se manter na escola; vitórias na Olímpiada de Matemática de Nova Iorque durante três anos seguidos; confiança e altas expectativas por parte dos pais dos alunos; e por fim, resultados em exames educacionais $56 \%$ melhores que a média da cidade de Nova Iorque (LEMES; SANCHES, 2016).

Quadro 1 - Apresentação dos princípios do aprendizado aplicados pela Quest to Learn

\section{Princípios da Quest to Learn}

1. Todos são participantes: todos devem participar e contribuir, alunos diferentes podem contribuir com inteligências diferentes;

2. Desafio: o desafio deve ser constante. $O$ aluno necessita de incentivo para resolver desafios complexos;

3. Aprendizado na prática: o aprendizado é ativo, alunos aprendem na prática testando e jogando;

4. Feedback imediato e contínuo: alunos conseguem ter feedback do seu desenvolvimento a partir de parâmetros de jogos como a pontuação;

5. Entender a falha como uma oportunidade: a falha é entendida como uma nova chance de aprender, assim como em um jogo, existe a opção de começar novamente;

6. Tudo está conectado: os alunos podem dividir seus conhecimentos e habilidades com outros por meio de comunidades, grupos e etc;

7. Sensação de estar jogando: a experiência de ensino deve engajar o aluno e dar suporte as suas ideias e criatividade.

Fonte: Adaptado de (LEMES; SANCHES, 2016).

Como a gamificação é um processo que pode seguir diferentes caminhos (HAMARI; KOIVISTO; SARSA, 2014) para atingir o objetivo principal, é necessário identificar as facilidades motivacionais geradas pelo processo, no contexto em que a gamificação está sendo aplicada, e alinhá-las com o objetivo da aplicação. Da mesma forma que, como para a Quest to Learn, a definição de princípios também é destacada por Fadel et al. (2014) e está fortemente relacionada ao alinhamento proposto por Hamari, Koivisto e Sarsa (2014). É relevante destacar que Fadel et al. (2014) propõem uma associação entre as características ou elementos da gamificação e seus respectivos princípios de aplicação. $\mathrm{O}$ Quadro 2 resume alguns desses elementos.

Quadro 2 - Apresentação das características da gamificação e suas respectivas definições

\begin{tabular}{|l|l|}
\hline Característica & Definição \\
\hline \multirow{5}{*}{ Campanha } & $\begin{array}{l}\text { Campanhas podem levar } \\
\text { os jogadores a uma } \\
\text { jornada. Pode se referir a } \\
\text { uma lista de tarefas, } \\
\text { ordenadas ou não, que } \\
\text { devem ser cumpridas para } \\
\text { obter-se uma recompensa. }\end{array}$ \\
\hline Cooperação & $\begin{array}{l}\text { Fundamental para a } \\
\text { construção de uma } \\
\text { comunidade forte. } \\
\text { Utiliza-se nessa aplicação } \\
\text { características do jogo } \\
\text { que permitam os } \\
\text { jogadores colaborar uns } \\
\text { com os outros. }\end{array}$ \\
\hline Forma de arte importante \\
para garantir que a \\
experiência gamificada \\
seja agradável.
\end{tabular}

Fonte: Adaptado de (FADEL et al., 2014).

Os autores evidenciam a necessidade da definição de princípios e entendimento da aplicação de cada característica proposta pela gamificação para que, então, o estado de flow seja atingido pelo usuário. $\mathrm{O}$ alinhamento dos princípios ao objetivo de aplicação da gamificação é fundamental também para uma 
correta fundamentação do processo de criação de regras e conteúdo do ambiente gamificado.

Fadel et al. (2014) alertam também sobre a importância da identificação de perfis ao se propor um ambiente gamificado e concluem que o modelo gamificado proposto pela plataforma potencializa as características da "Geração C", marcada por pessoas que adotam um tipo de comportamento social cada vez mais conectado. Ainda segundo os autores, o perfil dessa geração é caracterizado pelo compartilhamento, conectividade e colaboração.

Fadel et al. (2014), Fardo (2013) e Lemes e Sanches (2016) enxergam a educação como o campo com maior potencial a ser explorado pela gamificação. Entretanto, para Fardo (2013), mesmo com as demandas em relação às tecnologias, trazidas pelo século XXI, não houve mudanças consideráveis nas escolas e no ensino tradicional no contexto brasileiro. Lemes e Sanches (2016) relacionam esse cenário de poucas mudanças ao fato de haver poucos estudos e relatos de experiências gamificadas em língua portuguesa. É evidente que o tema caminha de forma devagar no cenário da educação no Brasil.

\section{O Modelo de Plataforma Gamificada por Silva e Bax (2017)}

Silva e Bax (2017) desenvolvem um ensaio teórico no qual discutem o tema da gamificação aplicada à educação em ambientes on-line, baseando-se em sete artigos principais. Segundo os autores, o modelo determina um conjunto de comportamentos esperados dos usuários, sendo cada tipo de participante resultado de um conjunto de comportamentos, tais como a frequência de utilização da plataforma; a busca pelo aprendizado por meio do conteúdo disponibilizado; a verificação do aprendizado, principalmente por meio de atividades; a interação com outros usuários; a busca pela superação de desafios e por recompensas; a autonomia na escolha do percurso a ser seguido para cumprir com os objetivos de aprendizado entre outros (SILVA; BAX, 2017).

Metodologicamente, os autores desenvolveram o modelo seguindo os seguintes passos: a) definição do objetivo do modelo proposto para a plataforma gamificada de educação on-line; b) identificação dos perfis de estudantes; c) inserção de diversão no ambiente; d) delineamento dos comportamentos-alvo; e) definição dos objetivos de design que contribuem e suportam os comportamentos-alvo (SILVA; BAX; 2017).

Os autores também apontam algumas limitações relacionadas à forma de aplicação das mecânicas e elementos da gamificação no contexto educacional. Destaca-se, neste contexto, o número restrito de mecânicas de jogos (como pontos, medalhas e painéis de liderança) aplicadas sem se levar em consideração os diferentes perfis de estudante.

\section{CONCLUSÕES}

Os resultados apresentados neste artigo evidenciam o potencial da gamificação como metodologia de ensino para superar dois grandes desafios enfrentados pelo Ensino em Engenharia de Produção: a contínua inserção de novas tecnologias e a necessidade de aproximação do ensino com a prática no cotidiano.

A utilização de jogos como metodologia de ensino em educação superior já é explorada em projetos de tecnologia nas áreas de inteligência artificial, redes neurais e deep learning, como no caso do Scratch ${ }^{\circledR}$. Adicionalmente, notou-se o emprego do jogo Production Line ${ }^{\circledR}$ como um método que foca na aprendizagem vivencial, desenvolvendo competências relacionados à tomada de decisão, gestão financeira e gerenciamento de recursos e de riscos. Nesse sentido, reforça-se a utilização da gamificação como metodologia capaz de adaptar o Ensino em Engenharia de Produção frente aos principais desafios e tendências em educação, contribuindo para a formação de profissionais mais capacitados, trabalhando aspectos reais e cotidianos em situações profissionais que podem ser simuladas ainda durante o processo de formação do estudante.

Ademais, este trabalho objetivou também explorar a gamificação aplicada pela escola nova-iorquina Quest to Learn. Evidenciou-se dois aspectos principais: a definição de 
princípios de gamificação; e o alinhamento entre os objetivos da aplicação e a metodologia. De forma complementar, identificou-se que o framework de gamificação de Silva e Bax (2017) contempla duas etapas que são consideradas essenciais por Fadel et al. (2014) e Hamari, Koivisto e Sarsa (2014) para que os benefícios da gamificação sejam atingidos na prática em contextos educacionais, sendo elas: a identificação de diferentes perfis de estudantes; e a etapa de associação entre objetivo de aplicação, comportamento-alvo de cada perfil e características da gamificação, nomeadas por Silva e Bax (2017) como "objetivos do design". Assim sendo, é notável a existência de aspectos em comum entre o framework proposto por Silva e Bax (2017) e a aplicação de gamificação realizada pela escola Quest to Learn, na qual estudantes e instituição atingiram diversos êxitos. Esses aspectos são: a definição de princípios de gamificação, referidos no modelo de Silva e Bax como objetivos do design; e o alinhamento entre objetivo de aplicação e metodologia a ser inserida.

Dessa forma, o presente artigo ressalta o potencial do framework proposto pelos autores, considerando todos os aspectos expostos e atentando-se às limitações previamente observadas, como forma de possibilitar um novo tipo de aplicação da gamificação, alinhado com as principais tendências de educação superior. A reflexão do atual cenário de utilização de gamificação em contextos educacionais, aliada à análise do ensaio teórico realizado por Silva e Bax (2017), permitiu elaborar a seguinte proposição para nortear trabalhos futuros: o framework de design de plataforma educacional gamificada proposto por Silva e Bax (2017) permite adaptação para outros contextos além da educação corporativa, inclusive no cenário do Ensino de Engenharia. Assim, tornar-se-á possível coletar dados em um novo contexto, aperfeiçoar a proposta do modelo e contribuir para o tema da gamificação na educação superior.

\section{REFERÊNCIAS}

AZEVEDO, A. A. D.; GONTIJO, T. S. Habilidades, competências e o perfil do profissional de Engenharia de Produção no sudeste brasileiro. Formação@ Docente, v. 9, n. 2, p. 96-109, 2017.

BECKER, A. S. et al. NMC Horizon Report: 2016 Higher Education Edition. Austin, Texas: The New Media Consortium, 2006.

BECKER, A. S. et al. NMC Horizon Report: 2017 Higher Education Edition. Austin, Texas: The New Media Consortium, 2017.

DA SILVA, R. R. L. et al. O uso de jogos e simulação como métodos alternativos de ensino em engenharia no Brasil: uma revisão bibliográfica. Revista ESPACIOS, v. 37, n. 5, 2016.

CARVALHO, F. T.; CUNHA JÚNIOR, J. J.; OLIVEIRA, O. G. Kanban SA: Serious game para ensino de gestão da produção. Revista de Ensino de Engenharia, v. 39, n. 2, 2019.

FADEL, L. M. et al. Gamificação na Educação. São Paulo: Pimenta Cultural, 2014.

HAMARI, J.; KOIVISTO, J.; SARSA, H. Does Gamification work? A Literature Review of Empirical Studies on Gamification. 47th Hawaii International Conference on System Science, 2014.

KAFAI, Y. The educational potential of electronic games: From games-to-teach to games-to-learn. Playing By The Rules, Cultural Policy Center, University of Chicago, Chicago, IL, 2001.

KAPP, K. M. The gamification of learning and instruction: game-based methods and strategies for training and education. San Francisco: Pfeiffer, 2012.

KAUFMANN, D. A. Reflection: Benefits of Gamification in online higher education. Grand Canyon University. Journal of Instructional Research, v. 7, 2018.

LEE, J. J.; HAMMER, J. Gamification in Education: What, How, Why Bother? 
Academic Exchange Quarterly, v. 15, p. 1-5, 2011.

LEMES, D.; SANCHES, M. H. Gamificação e Educação: Estudo de caso da Escola Quest to Learn. Simpósio Brasileiro de Jogos e Entretenimento Digital, 2016.

MALAN, D. J.; LEITNER, H. H. Scratch for budding computer scientists. ACM Sigcse Bulletin, v. 39, n. 1, p. 223-227, 2007.

MCGONIGAL, J. Reality is broken: why games make us better and how they can change the world. New York: The Penguin Press, 2011.

SAILER, M. et al. How gamification motivates: An experimental study of the effects of specific game design elements on psychological need satisfaction. Computers in Human Behavior, v. 69, p. 371-380, 2017.

SILVA, F. B. Implicações da gamificação no projeto de plataforma de educação on-line: um estudo de caso. Tese (Doutorado em Ciência da Informação) - Escola de Ciência da Informação, Universidade Federal de Minas Gerais. Belo Horizonte, p. 1-194. 2018.

SILVA, F. B.; BAX, M. P. Gamificação na educação online: proposta de modelo para a aprendizagem participativa. Encontros Bibli: revista eletrônica de biblioteconomia e ciência da informação, v. 22 , n. 50 , p. 144160, 2017.

\section{DADOS BIOGRÁFICOS DOS AUTORES}
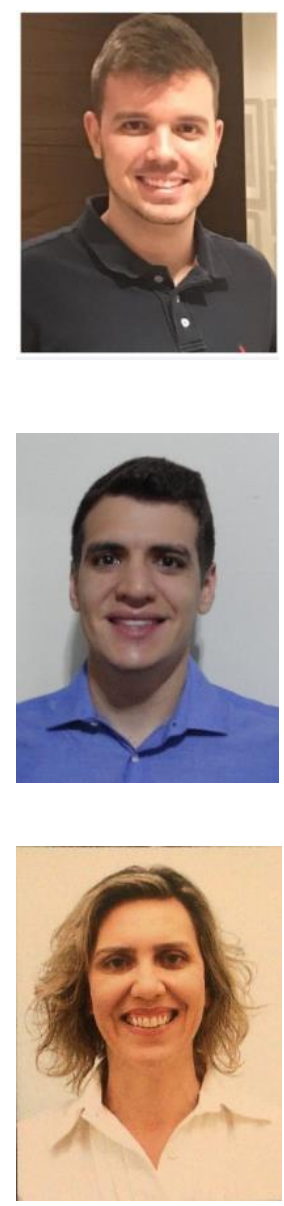

Lucas Bergman Aleixo Fóscolo de Moura - Graduação em Engenharia de Produção - PUC Minas (2019). Atuou como analista de inteligência de mercado e marketing nas empresas Vallourec e Heineken. Atualmente, é coordenador de marketing na Ambev, responsável pela cervejaria Wäls. Tem interesse em assuntos relacionados à empreendedorismo, inovação, gamificação e marketing.

Leonardo Assirio de Oliveira Bossi - Graduação em Engenharia de Produção PUC Minas (2020). Atuou na área de gestão de projetos e de inteligência de negócios na Offerwise. Tem interesse em assuntos relacionados à área de dados, inteligência de negócios, inovação e gamificação.

\footnotetext{
Paula Karina Salume - Graduada em Administração - PUC Minas (1996). Possui Especialização em Gestão Estratégica de Marketing - PUC Minas (1997); Especialização em Educação a Distância - PUC Minas (2011); Especialização em Gerenciamento de Projetos - PUC Minas (2013). É Mestre em Administração PUC Minas (2006) e Doutora em Administração - PUC Minas (2016). Professora e Pesquisadora do Departamento de Ciências Administrativas e Contábeis da Universidade Federal de São João Del-Rei. Foi professora e coordenadora na PUC Minas. Atuou como analista de planejamento e coordenadora de infraestrutura na Companha de Gás de Minas Gerais. Foi coordenadora e diretora administrativa da rede Coleguium de ensino. Tem interesse no ensino de empreendedorismo e gestão de projetos na engenharia.
} 